\title{
Editorial
}

\section{Galardones europeos a las buenas prácticas en seguridad y salud en el trabajo}

\section{European Good Practice Awards on Safety and Health at Work}

\author{
Belén Pérez Aznar', Pilar Casla Benito' \\ 1. Departamento de Relaciones Internacionales - Instituto Nacional de Seguridad y Salud en el Trabajo. Madrid. \\ España. \\ Correspondencia \\ Belén Pérez Aznar \\ Departamento de Relaciones Internacionales - Instituto Nacional de Seguridad y Salud en el \\ Trabajo \\ Madrid. España \\ Correo electrónico: bperezaz@insht.meyss.es
}

La Agencia Europea para la Seguridad y la Salud en el Trabajo organiza, con motivo del comienzo de cada Campaña sobre trabajos saludables, el Certamen de Galardones Europeos a las Buenas Prácticas como reconocimiento a las organizaciones que llevan a cabo acciones sobresalientes e innovadoras en materia de seguridad y salud en el trabajo.

Los Galardones Europeos a las Buenas Prácticas constituyen uno de los principales pilares de las campañas europeas que, cada dos años, la Agencia impulsa a lo largo de Europa y que son apoyadas por las instituciones que conforman la Red Española de Seguridad y Salud en el Trabajo.

La selección de ejemplos de buenas prácticas se realiza en dos fases. En una primera fase, se seleccionan las dos mejores candidaturas recibidas a escala nacional en cada Estado miembro (para cada una de las dos modalidades, de menos de 100 trabajadores, y de más de 100 trabajadores). En España, en este proceso de selección, participan los interlocutores sociales, la autoridad laboral de las comunidades autónomas, la Inspección de Trabajo y Seguridad Social y el Instituto Nacional de Seguridad e Higiene en el Trabajo (INSHT).

En una segunda fase, se seleccionan las mejores candidaturas a escala europea, que reciben el galardón en una Ceremonia de difusión mediática, organizada por la Agencia Europea. La pasada convocatoria de este certamen tuvo resultado exitoso para una microempresa española que obtuvo el Galardón Europeo, Protón Electrónica SLU.

Al margen de la competición europea, tanto el INSHT como la Red Española, colaboran cada año con la Agencia Europea en la difusión de los ejemplos de las empresas seleccionadas, como reconocimiento a su trabajo y a su contribución para la mejora de la seguridad y salud en el trabajo, logrando mediante esta difusión que otras empresas puedan conocer soluciones de éxito y, en muchos casos, adaptarlas a su contexto. En esta labor de difusión se invita a participar a medios de comunicación, ya que constituyen una pieza fundamental en el engranaje de la cultura preventiva y contribuyen a que otras empresas puedan conocer formas eficaces de realizar una gestión de la seguridad y la salud en el trabajo desde el liderazgo de la dirección y la participación de los trabajadores.

Durante el bienio 2012-2013, la Campaña Europea “Trabajando juntos para la prevención de riesgos” ha estado presente en más de 90 actividades (jornadas, seminarios, talleres, concursos, artículos, exposiciones, etc.) organizadas en España, gracias a la colaboración de numerosas instituciones que trabajan en el ámbito de la prevención de riesgos laborales. A través de estas acciones de apoyo a la campaña se ha logrado subrayar la necesidad de que directivos y trabajadores aúnen sus esfuerzos en materia de prevención de riesgos 
laborales, incidiendo de una manera especial en las ventajas que supone esta implicación conjunta mediante casos prácticos.

Gracias a los ejemplos seleccionados en la pasada edición de los Galardones Europeos a las Buenas prácticas hemos tenido ocasión de conocer experiencias de éxito, que demuestran que el liderazgo de la dirección y la participación de los trabajadores en materia de seguridad y salud en el trabajo es hoy una realidad en aquellas empresas españolas que son más seguras, saludables y productivas. Les animamos a conocer los detalles de estas iniciativas sobresalientes en materia de seguridad y salud en el trabajo, que se presentan a continuación.

El próximo año habrá una nueva edición de Galardones Europeos a las Buenas Prácticas, enmarcada en la Campaña Europea 2014-2015 sobre riesgos psicosociales, en la que se buscarán las soluciones prácticas más destacadas e innovadoras para gestionar activamente el estrés y los riesgos psicosociales en el trabajo. Las bases de esta nueva convocatoria se publicarán en el primer cuatrimestre de 2014, esperando recibir el mayor número posible de candidaturas de empresas y organizaciones españolas que puedan optar al reconocimiento europeo a su buen hacer.

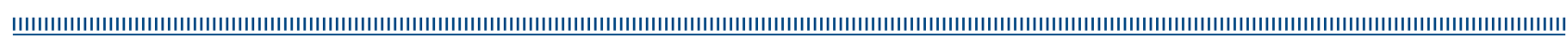

